\title{
Challenges and opportunities for effective contraceptive counselling in a low- resource setting: insights from a qualitative study in western Kenya
}

\author{
Francis Obare ${ }^{1}$, George Odwe ${ }^{1}$, \& John Cleland ${ }^{2}$ \\ ${ }^{1}$ Population Council, Nairobi, Kenya \\ ${ }^{2}$ Faculty of Epidemiology and Population Health, London School of Hygiene \\ and Tropical Medicine, UK \\ Email: fonyango@popcouncil.org
}

\begin{abstract}
Background: Evidence from parts of sub-Saharan Africa shows variations in contraceptive counselling by type of sector (public or private) and socio-economic background of clients. There is, however, limited understanding of the nature of interactions between family planning service providers and their clients that could ultimately influence the quality of counselling received by different sub-groups of clients. This paper explores the challenges and opportunities for effective contraceptive counselling in a low-resource setting in Kenya.

Data Source and Methods: Data are from a qualitative study that was conducted in 2018 among 42 women of reproductive age who participated in a longitudinal research project in Homa Bay County. The data were analysed using an exploratory inductive content analysis approach.

Results: The findings showed that challenges to effective contraceptive counselling were both providerand client-related. Provider-related challenges included workload, lack of competence in contraceptive counselling in general and on side effects in particular, and negative attitudes towards specific methods. Client-related challenges entailed presenting with fixed minds, lack of awareness of what to expect during interactions with providers, passive involvement in consultation process, and familiarity with service providers.

Conclusion: Some of the challenges could be addressed by information, education and communications interventions to empower clients to demand quality services as well as skills updates for providers to improve their capacity to respond to clients' needs.
\end{abstract}

Keywords: Contraceptive counselling; challenges and opportunities; low-resource setting; women of reproductive age; western Kenya

\section{Introduction}

Global family planning initiatives such as Family Planning 2020 (FP2020) and national programs aim to achieve universal access to effective contraception in low and middle-income countries (LMICs) as a means of reducing unintended pregnancies and the associated negative health and socio-economic consequences such as unsafe abortion, miscarriage, psychosocial health problems, and socio-economic deprivation (FP2020 Rights and Empowerment Working Group, n.d.). Achieving universal access to contraception in settings such as sub-Saharan Africa (SSA) is, however, hampered by a number of challenges including human resource, socio-economic and cultural constraints as well as limited availability of services and poor quality of care (Blackstone et al. 2017; Mutumba et al. 2018; Olakunde et al. 2019; Schivone and Blumenthal 2016). Global guidelines on provision of family planning services consider healthcare workers as key to ensuring acceptable quality of services (Jaccard and Levitz 2013; $\mathrm{WHO} / \mathrm{RHR}$ and JHBSPH/CCP 20I8). In particular, family planning service providers are called upon to counsel clients to enable them make voluntary and informed choices regarding their preferred methods, and to support them in solving any problems that arise when using their chosen methods (laccard and Levitz 20I3; WHO/RHR and JHBSPH/CCP 20I8). There is evidence that quality contraceptive counselling improves uptake and continuation of contraception and is therefore likely to contribute to the achievement of optimal protection against unintended pregnancy (Cavallaro et al. 2019; Chakraborty et al. 2019; Dehingia et al. 2019; Liu et al. 20I8; Tumlinson et al. 2015).

Effective counselling requires service providers to develop close and trusting relationships with clients, and to engage them in shared decision-making by determining and responding to their needs and 
preferences (Dehlendorf et al. 2014; WHO/RHR 2005; WHO/RHR and JHBSPH/CCP 20I8). The World Health Organization (WHO) recommends different counselling tasks for service providers depending on the type of family planning client, that is, whether they are new clients with and without a method in mind or returning clients with and without challenges (WHO/RHR 2005; WHO/RHR and JHBSPH/CCP 20I8). In particular, for a new adopter with a method in mind, providers are required to ensure that the client understands how to use the method, support the client's choice if she is medically eligible to use the method, provide information on how to use the method, and discuss how to cope with any side effects (WHO/RHR 2005; WHO/RHR and JHBSPH/CCP 2018). For a new adopter with no method in mind, providers should describe all available methods without overloading the client with information, help the client reach a decision, support the client's choice, provide information on how to use the chosen method, and discuss how to cope with any side effects (WHO/RHR 2005; WHO/RHR and JHBSPH/CCP 20I8). Returning clients with no problems should be asked how they are doing and whether they have any questions about the method while those returning with problems should be guided and a joint decision reached on whether to continue with or without drug therapy or to switch to an alternative method (WHO/RHR 2005; WHO/RHR and JHBSPH/CCP 20I8).

Understanding clients' needs and giving appropriate information and services are therefore key to quality provision of contraceptive services. Providing appropriate information to clients is one of the six components of quality of care proposed by Bruce (1990). Other components include ensuring that services are convenient and acceptable to clients, availability of a variety of methods to choose, technical competence of providers, good relations between providers and clients, and having mechanisms for follow-up (Bruce 1990). At a minimum, an approach that has been used to assess quality of contraceptive counselling is the method information index (MII) that entails determining whether clients are informed about other methods, possible side effects of their chosen method, and what to do if they experience side effects (Chakraborty et al. 2019; Chang et al. 2019). The recommendations and approaches to quality contraceptive counselling therefore place great emphasis on the role of service providers in ensuring effective counselling of family planning clients. However, quality contraceptive counselling in lowresource settings may be hampered by availability of trained service providers and other health systems challenges such as inadequate infrastructure and unreliable commodity supply chain.

Besides provider availability and skills, studies in SSA show that the quality of contraceptive counselling varies by type of sector (public or private) and socio-economic background of clients. Quality of contraceptive counselling is, for instance, higher among women with high levels of education or from wealthy households than among those with low levels of education or from poor households (Hrusa et al. 2020; Tessema et al. 2016). Moreover, whereas quality of contraceptive counselling is higher in public than in private health facilities, clients in the private sector report higher levels of satisfaction with services than those in the public sector (Hrusa et al. 2020; Tessema et al. 2016). In spite of the evidence showing variations in contraceptive counselling, there is limited understanding of the nature of interactions between family planning service providers and their clients that could ultimately influence the quality of counselling received by different sub-groups of clients. It could, for instance, be that women with high levels of education or from wealthy households are likely to be aware of their rights and to ask for more information about methods compared to those with low levels of education or from poor households. Similarly, it could be that clients who seek services from the private sector secretly use contraceptives, value privacy and the need for quick services, and thus derive their satisfaction from the process of obtaining services rather than information given (Hutchinson et al. 20I I; Keesara et al. 20I5). In this paper, we use qualitative data from a study that was nested within a longitudinal research project in a rural setting in Kenya to examine challenges and opportunities for effective contraceptive counselling based on women's interactions with service providers when obtaining family planning services.

\section{Study context}

The study was conducted in Homa Bay County located in western part of Kenya along the shores of Lake Victoria. In terms of demographic composition, females comprise $50 \%$ of Kenya's 47 million people, and $52 \%$ of Homa Bay's I.I 3 million people (KNBS 2019). Women of reproductive age (I5-49 years), on the other hand, comprise $50 \%$ of Kenya's and $46 \%$ of Homa Bay's female population (KNBS 2019).

Kenya has made remarkable progress in the uptake of modern methods of contraception, with use of the methods among currently married women almost tripling over a period of two and a half decades, from $18 \%$ in 1989 to $53 \%$ in 2014 (NCPD and IRD/Macro Systems Inc. 1989; KNBS et al. 20I5). However, the remarkable progress masks wide disparities in contraceptive use between counties. 
Use of modern methods among currently married women ranges from 2\% in Mandera County in North Eastern region to $76 \%$ in Kirinyaga County in the Central region of the country (KNBS et al. 2015). Homa Bay County falls between the two extremes with a modern contraceptive prevalence rate of $46 \%$ (KNBS et al. 2015). Similar to contraceptive use, the total fertility rate (TFR) in Kenya declined from 6.7 children per woman in 1989 to 3.9 children per woman in 2014 (NCPD and IRD/Macro Systems Inc. 1989; KNBS et al. 2015). There are also marked variations in TFR between counties, ranging from 7.8 children per woman in Wajir County in North Eastern region to 2.3 children per woman in Kirinyaga County, with Homa Bay County having a TFR of 5.2 children per woman (KNBS et al. 20I5).

Although health is a devolved function (that is, counties manage their own health systems), the national government plays an important role in formulating policies, standards and guidelines for health service provision in both public and private sectors in the country (MOH 20I8). With respect to family planning, the national guidelines recognize the importance of effective counselling in promoting uptake and continuation of methods, and the role of service providers in ensuring quality counselling of clients $(\mathrm{MOH}$ 20l8). However, similar to other LMICs, the public health sector in the country faces human resource challenges that may hamper effective counselling of family planning clients. These include limited availability of essential cadres of staff, uneven distribution of existing cadres across geographic regions and levels of health service delivery, and low productivity due to heavy workload and poor remuneration (MOH 20l3). The private sector, on the other hand, comprises $49 \%$ of health facilities in the country, that range from small private clinics and dispensaries to big for- and not-for-profit hospitals (MOH 2014). The diverse nature of the sector implies that providers who offer high quality services may not be accessible to the poor while those whose services are accessible to the poor may lack the technical competence to effectively counsel clients (Mills et al. 2002; Patouillard et al. 2007).

\section{Methods}

The data used in this paper are from a qualitative study that was nested within a longitudinal research project implemented in Homa Bay County of Kenya between 2016 and 2018. Detailed description of the longitudinal research project is provided elsewhere (Machiyama et al. 2017). The qualitative study was conducted in 2018 during the final round of the research project. It involved in-depth interviews with 42 women of reproductive age (I5-39 years at the time of recruitment into the longitudinal research 5189 project) who discontinued injectables or implants the two dominant women-controlled methods in the project site - between the first two rounds of the research project (2016 and 20I7). The data showed that $40 \%$ of the women who were using a method at the time of recruitment into the study had discontinued use by the second round, with the level of discontinuation of injectables and implants being $42 \%$ and $20 \%$, respectively, which were higher than the national average of $31 \%$ for all methods, $31 \%$ for injectables, and $8 \%$ for implants.

The purpose of the qualitative study was to understand contraceptive use and provision practices that are likely to influence the high levels of method discontinuation in the study setting. Participants were purposively identified based on the method they had discontinued ( $2 \mathrm{I}$ for implants and $2 \mathrm{I}$ for injectables), major reason for discontinuation (side effects/health concerns, wanting to become pregnant, contraceptive failure, infrequent sex, desire for a more effective method, inconvenience of use, and husband disapproval), and the sub-county where the research project was undertaken (Ndhiwa, Rachuonyo North and Rachuonyo South). In order to ensure fair representation of views from all study sites for all reasons of discontinuation, one participant who discontinued using either method for each of the seven reasons was targeted for interview in each subcounty.

Interviews with the selected participants were conducted a few days after they completed the final round of survey interviews by two research assistants with training in qualitative data collection. Participants were asked about their experiences with the following: (i) access to information on the method before initiating use; (ii) interactions with service providers when obtaining the method; (iii) experiences with using the method, including side effects; (iv) actions taken after discontinuing use, including switching to another method or discontinuing use altogether; and (v) attitudes towards the method in particular and contraception in general based on experiences with use. This paper focuses on participants' description of their interactions with service providers when obtaining methods to identify the challenges and opportunities for effective contraceptive counselling.

The interviews were conducted in the local language (Dholuo), were audio-recorded with the consent of participants, and lasted on average 42 minutes. They were then transcribed and translated into English by the research assistants who conducted the interviews but without back-translation to determine if any meaning was lost in the process. The data were analysed using an exploratory inductive content analysis approach, which entailed reading and 
re-reading the transcripts by the lead author to identify challenges and opportunities that emerged from participants' interactions with service providers. The identified themes were then shared with the coauthors who had also read the transcripts for concurrence. Specific quotes from the interviews are used to support the key insights from such interactions. The quotes are identified by the age of the women at the time of recruitment into the study, the method they discontinued between the first two rounds, and the reason for discontinuation.

Written informed consent was obtained from all participants before conducting the in-depth interviews. The study was granted ethical approval by the Observational/Interventions Research Ethics Committee of London School of Hygiene and Tropical Medicine, the Institutional Review Board of the Population Council, and Kenyatta National Hospital/University of Nairobi Ethics and Research Committee. In addition, the National Commission for Science, Technology and Innovation granted the research permit for conducting the study in Kenya.

\section{Results}

\section{Characteristics of participants}

Seven of the 42 participants interviewed were aged below 20 years at the time of recruitment into the study, 13 were aged 30 years or older, while the remainder were between 20 and 29 years old. Twelve participants had no formal education or had incomplete primary level education, 15 had complete primary education and another 15 had secondary or higher levels of education. Eight participants had only one living child at the time of recruitment, 23 had three or more children while the remainder had two children. Participants were evenly distributed across the three sub-counties where the research was undertaken (I 4 participants in each sub-county).

\section{Challenges to effective contraceptive counselling}

The narratives indicate that challenges to effective contraceptive counselling were both provider- and client-related.

\section{a) Provider-related challenges}

Provider-related challenges pertained to workload, competence, and attitudes. Some participants who sought care from public health facilities reported long queues and heavy workload that did not allow service providers to spend sufficient time with clients to give adequate information about the methods provided. Others who obtained methods from small private clinics or pharmacies where there were no long queues reported that the encounters were like a business transaction whereby the client stated what she needed, the provider told her the cost, the client paid, the provider offered the method, and that marked the end of the interactions. Participants who sought services from private outlets did so because they were secretly using contraceptives and wanted quick and confidential services, the facilities were near, or there were stock-outs of commodities in the public facilities. It was not clear from the interviews why private providers did not give adequate information on the methods they offered clients despite having the time to do so given the light workload. However, one participant reported being told by a private provider that he only gives what clients ask for and does not propose to clients to use what they may be uncomfortable with. This suggests that such providers fear driving away clients and thus affecting business especially if the methods they propose to clients negatively affect them. The following excerpts highlight some of these experiences:

She asked about my last date of monthly period; she asked about the dates I normally experience menstruation... Nothing else...We did not have much discussion over it because there were so many clients in the queue waiting to be served. We did not talk much. In fact, we had no discussion on the advantages or disadvantages of implant. She only told me that implant is good but did not elaborate much on that (18-year old; implant - side effects).

These people don't bother to ask questions. Such questions are only asked at the District Hospital. These private providers will ask you for money and once you have paid, they inject you and that's the end of your business with him... They will only ask your name, if you are on your periods and if you have any child and the story ends there but not anything as deep as what you people are asking (23-year old; injectable - side effects)

The inability of private providers to adequately counsel clients on contraceptive methods offered could also be due to lack of technical competence in contraceptive counselling. However, this also affected providers in the public sector, especially with respect to the completeness of information given to family planning clients. Most participants who obtained methods from public health facilities and big private hospitals reported being told about the advantages of family planning and all of them narrated those advantages. Participants also reported being informed about other available methods in such facilities, including some of those who went with a preferred method in mind. Nonetheless, only seven of the 42 participants reported being informed about the side effects of the methods they obtained, 
including heavy or irregular bleeding, headache, and nausea. Some participants who were not informed about side effects reported simply being told to return to the facilities if they experienced problems without being told what those problems were. This suggests that most providers had challenges counselling clients on side effects of the methods, and tended to focus only on the benefits of contraception. Some of these experiences are exemplified in the following quotes:

It was upon you to experience a problem with a method and then decide whether to discontinue the method or not. They normally don't talk about unpleasant side effects of these methods because this might discourage people from using them (29-year old; implant - out of stock).

We were advised to return to the facility in case of any side effects although I did not experience much problems with it...they did not give us information on the possible side effects (32-year old; implant - became pregnant while using).

Provider attitudes manifested in the tendency of some providers to dissuade clients from using specific methods based on their negative beliefs about those methods. One participant who wanted injectables was dissuaded from it because the provider told her that it would affect production of milk for her baby, and was instead given implant. Another participant who wanted injectables was convinced to use implant because, according to the provider, it was the method that most women were using at that point in time. A third participant reported being dissuaded from using intra-uterine device by the provider because it could cause cancer, and even from using the three-year implant because the duration was short. She was instead convinced to use five-year implant. Another participant reported that she was told by the provider that injectable is not generally good because it may delay conception when one stops using it and that implant is a better option. The narratives further showed that during outreach, providers tended to emphasize the method being promoted during such activities even if they informed clients about other methods. Similarly, in health facilities where providers talked about available methods, they only focused on those that were available at the time and left out those that were out of stock. In such circumstances, it is likely that clients may not be given sufficient information about other methods in a manner that would enable them make informed and voluntary decisions regarding the method to use. These biases could also be driven by targets set for providers regarding uptake of specific methods, especially during outreach activities. The $519 \mid$ following quotes are examples of some of these experiences:

At first, I told her to provide me with injectables but she declined. She told me the child was still young and using injectables could result in low milk production hence starving the child (22-year old; implant - side effects).

In fact, nothing was explained there...I was only advised to avoid coil [intra-uterine device] because the method could cause cancer in case I used it... He advised me to use five-year implant because it lasts for a longer duration... He also declined to give me three-year implant citing shorter duration. So, that is the reason why I was given five-year implant (24-year old; implant wanted to become pregnant).

\section{b) Client-related challenges}

Client-related challenges included presenting with fixed minds regarding specific methods, lack of awareness of what to expect during interactions with providers, passive involvement in the consultation process, and familiarity with service providers. Whereas clients visiting health facilities with fixed minds regarding specific methods pose counselling challenges to service providers, the narratives showed that this should not necessarily be the case. Some of the participants who visited facilities with their minds fixed on specific methods reported that providers still informed them about other methods and the side effects of their chosen methods. This contrasts the experiences of some clients in private health facilities and pharmacies who presented with fixed methods in mind and the interactions were mostly business-oriented, with the client stating her needs, the provider quoting the price, and the client making the necessary payment before being given the service. This suggests that as long as providers are adequately equipped to effectively counsel clients, those presenting with fixed minds regarding specific methods should not pose a challenge to effective counselling on their chosen method, the associated side effects, and what to do in case of side effects. As one participated narrated,

She asked me about the method that I wanted to use. I told her, "Me, I want to use depo." Then she asked me why I preferred using depo then I told her that depo is the method I have chosen because I think it can be fine with me...She then told me about available family planning methods including implants, coil and that one of depo. She told me of condom as well...She told me that there are some unpleasant side effects that I may experience when using depo...like failure to menstruate completely, irregular menses, and http://aps.journals.ac.za 
after listening to her, I told her, "Just inject me with depo because I have fear for implants" (19year old; injectable - wanted to become pregnant)

The interviews further revealed that some participants were not aware of the kind of information they should expect from providers when obtaining family planning methods. In some cases, participants ended up asking the interviewers if providers are supposed to give clients the kind of information they were being asked if they received, or if providers are supposed to give any additional information beyond what they reported. Related to lack of awareness of what to expect during interactions with providers was passive participation in the consultation process. The narratives showed that apart from providing the necessary individual details (such as age, marital status, number of children, and date of last menstrual period), few participants asked for information on the methods they received which providers did not spontaneously mention even when the provider gave them an opportunity to ask questions. The reasons the participants gave for not asking questions were that they were using a method for the first time and trusted everything the provider told them, they were in a hurry to attend to other matters, they arrived late for the consultation, and that in cases where they were being given information as a group, nobody else in the group asked questions. The following quotes exemplify some of these experiences:

Personally, I was not taught anything given that I had gone late; maybe those who were there earlier were taught. I just went, gave my card and had my injection and came back home (28-year old; injectable - side effects).

I was told if I wanted it [implant] removed before they [outreach providers] come back, it can be done in any hospital provided it's not a nurse or doctor on attachment because they can do more harm than good...I did not ask how because for a first-time user, you just agree with everything being said since you don't know (33-year old; implant - side effects).

Clients' familiarity with providers, whether in the private or public sector, presented another challenge to effective contraceptive counselling. Some participants reported that providers already knew them from previous interactions when they sought maternal and child health or family planning services, and in the case of private providers, because they were their neighbours or family acquaintances. In such cases, participants reported that providers already knew what they wanted, and provided the services without giving much information about the methods. Whereas it could be that some of the clients were on return visits and had no problems with the method they were using, according to the global recommendations on counselling of such clients, providers should determine how they are doing with the method and give advice accordingly. However, the narratives suggest that this was not the case as shown by the following excerpts:

This is someone who knows me well... When you go in, you inquire if injectable is available; then he tells you the price and then you get injected; there is no discussion that takes place (19-year old; injectable - wanted to become pregnant).

They know I am from the locality...they don't always tell me those things [provide information on methods]. They know me so they don't even tell me. They want to assume that I know (32year old; injectable - wanted to take a break).

\section{Opportunities for effective contraceptive counselling}

Some of the challenges to effective contraceptive counselling of clients also provide opportunities for improving the provision of information on methods. For instance, in order to cope with human resource challenges and heavy workload in the public sector, providers resort to giving information to clients in groups before commencing provision of methods to individual clients. A number of participants mentioned being given information in groups, as exemplified in the following quote:

The provider told us that the method is not effective one hundred percent, so she advised us to take precaution when using the method because we could still become pregnant... This information was given in a group for all women who went for family planning on that day (19-year old; injectables - wanted to become pregnant).

Such group sessions provide opportunities for giving key information on available methods, the advantages and side effects of each of the available methods, and what to do in case of side effects. Providers can also use the opportunity to encourage clients to ask questions either during the group sessions or when they are individually being given the methods after the group sessions. In that way, the time taken with individual clients is greatly reduced and at the same time, clients receive the key pieces of information regarding their preferred methods.

Another opportunity for effective contraceptive counselling arises from clients who present with preferred methods in mind. The narratives showed that in most cases, such clients already received 
information on methods from their peers (although some of that information was not entirely complete or correct) or when they sought other maternal and child health services. As previously mentioned, some providers used the opportunity of clients presenting with preferred methods in mind to give information on available methods, their side effects, and what to do in case of side effects although the practice was not common across providers. The advantage to providers is that counselling such clients may take a relatively shorter time compared to those who are naive about methods. The quote below highlights a case of a participant who went to the facility with a preferred method in mind but was still given information on possible side effects of her preferred method:

Yes, I wanted to use injectable as a method of family planning when I went to the facility at that particular time. I went there already with a method in mind. I just told them, "I want injectables"...I was told that I may experience headache, heavy bleeding and even irregular monthly period when using the method; those were some of the problems they mentioned one might experience while using the methods but they said it is important to return to the facility for further medical assistance from the provider although they said headache and fever are normal sicknesses that are expected in life (35-year old; injectables - partner disapproval).

The third opportunity for effective contraceptive counselling emanates from client familiarity with service providers. Such familiarity should ideally provide opportunities for individualized contraceptive counselling whereby clients consult providers when they need more information and providers support clients when they experience problems with methods beyond the clinic setting.

\section{Discussion}

The findings of this paper show that challenges to effective contraceptive counselling in the setting where the study was conducted are both providerand client-related. Besides workload and biased attitudes towards certain methods, the findings show that providers tended to emphasize the benefits of contraception and few talked about side effects of specific methods when counselling clients. This is consistent with findings from a study in Ghana which found that providers rarely gave clients information on side effects of methods (Klu 2019). Failure to provide information on side effects and what to do implies that clients are left psychologically unprepared when they experience challenges with the methods, and are likely to resort to 5193 discontinuation as a solution to those challenges. Contraceptive counselling was even more limited in private clinics and pharmacies. The Kenya Ministry of Health undertakes contraceptive technology updates from time to time whenever necessary to equip providers in the public sector with skills on new and emerging family planning information and methods. Private sector providers, on the other hand, rely on programmes implemented by different non-state actors, which usually focus on specific geographic areas of the country. Such opportunities could be used to improve the capacity of providers in counselling on contraceptives generally (including addressing negative attitudes towards some methods) and on side effects in particular.

Client-related challenges to effective contraceptive counselling entailed presenting with fixed minds regarding specific methods, lack of awareness of what to expect during interactions with service providers, passive involvement in the consultation process, and familiarity with service providers. The extent to which these challenges limit effective contraceptive counselling may be influenced by clients' socio-economic background such as level of education and household wealth status. Studies in other SSA settings, for instance, show that women with high levels of education or from wealthy households are more likely to receive quality contraceptive counselling than those with low levels of education or from poor households (Hrusa et al. 2020; Tessema et al. 2016). This also reflects patterns in contraceptive use in the region, which is higher among women of high than those of low socio-economic status (Anasel and Mlinga 2014; Blackstone et al. 2017; Nonvignon and Novignon 20I4; Unumeri et al. 20I5). This suggests that better educated and wealthy women are not only likely to demand services but also good quality care. The findings of this paper further show that the extent to which the challenges limit effective contraceptive counselling varies by individual providers as some providers still offered the requisite information to clients who presented with preferred methods in mind. These findings suggest that client-related challenges to effective contraceptive counselling could be addressed by information, education and communications interventions aimed at enhancing the capacity of clients to demand quality services, as well as skills updates to improve the technical capacity of providers to effectively respond to clients' needs. Use of job aids such as flip charts and counselling cards could also help improve counselling (Population Council 2015). In addition, given high penetration of mobile phones, automated messages to users could help support clients who are on contraception and thus improve continuation.

http://aps.journals.ac.za 
The penetration of mobile phones and other digital technologies further provides avenues for exploiting some of the opportunities for effective contraceptive counselling identified in this study, including client familiarity with service providers and preference for specific methods based on information obtained from other sources. In particular, through digital technology, providers can leverage the rapport created with clients to offer individualized counselling and support to those on contraception beyond the clinic setting. In addition, providers can leverage such channels to offer accurate information on family planning to women who may have acquired knowledge of contraception from unqualified sources. Group counselling sessions used by providers in the public sector to cope with human resource challenges, on the other hand, provide opportunities for building the confidence of clients to be active rather than passive participants in the consultation process. Leveraging these opportunities could ultimately contribute to the realization of the principles of client-centred approach to family planning programming which emphasize support, respect and autonomy of clients (Diamond-Smith et al. 20I8; Walle and Woldie 2017).

In spite of the opportunities for improving contraceptive counselling in low-resource settings identified in this paper, there are likely to be persistent health systems challenges. For instance, high provider workload, which is occasioned by limited human resources, is likely to persist due to lack of adequate finances for hiring and retaining appropriate cadres of staff as well as other competing development priorities (MOH 20I4). This is likely to affect effective contraceptive counselling of clients as envisioned in both the global and national guidelines for provision of family planning services $(\mathrm{MOH} 2018$; WHO/RHR and JHBSPH/CCP 2018). Improving quality of contraceptive counselling in the private sector is also likely to be a persistent challenge. Studies show that the private sector performs better than the public sector in terms of the process of providing care (such as quick and confidential services) but not in the technical components of quality of care (Hutchinson et al. 20I I; Keesara et al. 2015). Although the Kenya Government formulates policies, standards and guidelines for health service provision in the country, it does not control the operations of private health facilities. Efforts to improve technical competence of private providers therefore depend on uncoordinated interventions implemented by various non-state actors in different parts of the country. It is not possible under such circumstances to determine the impact of the interventions on improving contraceptive counselling among private providers in the country. However, in urban areas, competition between private providers might stimulate higher quality of care unlike in rural areas where clients have a limited choice of providers.

There are certain limitations that may influence the findings of this paper. The qualitative study was based on purposively identified sub-set of women who discontinued either injectables or implants between the first two rounds of the longitudinal study. Their experiences when obtaining the methods may not be representative of all women, including those who did not discontinue use, those who used other methods, and those in urban settings. There is also the potential for recall bias as participants were asked to report on experiences that occurred in the past. In addition, there was no back-translation of the transcripts into the language of interview which limits the ability to determine if some meanings were lost in the process of translation into English.

\section{Conclusion}

Both provider- and client-related challenges limit effective contraceptive counselling in the setting where the study was conducted, and is likely to be so in similar contexts. Some of the challenges could be addressed by information, education and communication interventions to empower clients to demand quality services as well as skills updates for providers to improve their capacity to respond to clients' needs. However, some challenges such as provider workload and improving the technical competence of private providers are likely to persist due to lack of adequate financial resources for hiring healthcare workers, competing development priorities, and absence of proper coordination of different health sector players. Nonetheless, the penetration of digital technology coupled with opportunities created through provider-client interactions could contribute to overcoming some of the challenges to effective contraceptive counselling of clients.

\section{Acknowledgement}

This study was supported by UKaid from the Department for International Development (DfID) through STEP UP (Strengthening Evidence for Programming on Unintended Pregnancy) Research Programme Consortium. We acknowledge participants who volunteered information for the study, and Evans Omulo and Lydia Mauko who conducted the interviews. The opinions expressed in the paper are, however, solely those of the authors and do not necessarily reflect the views of the funding agency or STEP UP partners. 


\section{References}

Anasel, M.G. and Mlinga, U.J. 2014.

"Determinants of contraceptive use among married women in Tanzania: policy implication." African Population Studies 28, (2): 978-988.

Blackstone, S.R., Nwaozuru, U. and Iwelunmor, J. 2017. "Factors influencing contraceptive use in sub-Saharan Africa: a systematic review." International Quarterly of Community Health Education 37, (2): 79-9l.

Bruce, J. 1990. "Fundamental elements of the quality of care: a simple framework." Studies in Family Planning 2I, (2): 6I-9l.

Cavallaro, F.L, Benova, L., Owolabi, O.O., and Ali, M. 2019. "A systematic review of the effectiveness of counselling strategies for modern contraceptive methods: what works and what doesn't?" BMJ Sexual and Reproductive Health 0: I-16. doi: I0.1 I 36/bmjsrh-2019-200377.

Chakraborty, N.M., Chang, K., Bellows, B., Grépin, K.A., Hameed, W., Kalamar, A., Gul, X., Atuyambe, L. and Montagu, D. 2019. "Association between the quality of contraceptive counseling and method continuation: findings from a prospective cohort study in social franchise clinics in Pakistan and Uganda." Global Health: Science and Practice 7, (I): 87-102.

Chang, K.T., Mukanu, M., Bellows, B., Hameed,

W., Kalamar, A.M., Grépin, K.A., Gul, X. and Chakraborty, N.M. 2019. "Evaluating quality of contraceptive counseling: an analysis of the Method Information Index." Studies in Family Planning 50, (I): 25-42.

Dehingia, N., Dixit, A., Averbach, S., Choudhry,

V., Dey, A., Chandurkar, D., Nanda, P., Silverman, J.G. and Raj, A. 2019. "Family planning counseling and its associations with modern contraceptive use, initiation, and continuation in rural Uttar Pradesh, India.” Reproductive Health 16, (I78): III.

Dehlendorf, C., Krajewski, C., and Borrero, S.

20I4. "Contraceptive counseling: best practices to ensure quality communication and enable effective contraceptive use." Clinical Obstetrics and Gynecology 57, (4): 659-673.

Diamond-Smith, N., Warnock, R., and

Sudhinaraset, M. 2018. "Interventions to improve the person-centered quality of family planning services: a narrative review." Reproductive Health 15, (144): I-I7.

FP2020 Rights and Empowerment Working

Group (n.d.) Family Planning 2020: Rights and Empowerment Principles for Family Planning. Washington, $\quad$ DC: $\quad$ FP2020; https://www.familyplanning2020.org/sites/default/f
iles/FP2020_Statement_of_Principles_I Ix I7_EN 0922 1 5.pdf (Accessed: July 20 I9).

Hrusa, G., Spigt, M., Dejene, T. and Shiferaw, S.

2020. "Quality of family planning counseling in Ethiopia: trends and determinants of information received by female modern contraceptive users, evidence from national survey data, (20142018)." PLoS ONE 15, (2):e02287/4 https://doi.org//0.|37|/journal.pone.02287|4

Hutchinson, P.L., Do, M. and Agha, S. $201 \mathrm{I}$.

"Measuring client satisfaction and the quality of family planning services: a comparative analysis of public and private health facilities in Tanzania, Kenya and Ghana." BMC Health Services Research II, (203): I-I7.

Jaccard, J and Levitz, N. 20I3. "Counseling adolescents about contraception: towards the development of evidence-based protocol for contraceptive counselors." Journal of Adolescent Health 52: S6-SI3.

Keesara, S.R., Juma, P.A. and Harper, C.C. 2015. "Why do women choose private over public facilities for family planning services? A qualitative study of post-partum women in an informal urban settlement in Kenya." BMC Health Services Research 15, (335): I-8.

Klu, D. 2019. "Components of contraceptive counseling postpartum women receive during antenatal and postnatal health care services: a qualitative approach.” Journal of Gynecological Research and Obstetrics 5, (2): 048-054.

KNBS (Kenya National Bureau of Statistics). 2019. 2019 Kenya Population and Housing Census, Vol. I \& III. Nairobi: KNBS.

KNBS (Kenya National Bureau of Statistics), Ministry of Health (MOH), National AIDS Control Council (NACC), Kenya Medical Research Institute (KEMRI), and National Council for Population and Development (NCPD). 2015. Kenya Demographic and Health Survey 2014. Nairobi: KNBS, MOH, NACC, KEMRI and NCPD.

Liu, J., Shen, J. and Diamond-Smith, N. 2018.

"Predictors of DMPA-SC continuation among urban Nigerian women: the influence of counseling quality and side effects." Contraception 98: 430-437.

Machiyama, K., Casterline, J.B., Mumah, J.N., Huda, F.A., Obare, F., Odwe, G., Kabiru, C.W., Yeasmin, S. and Cleland, J.C. 20I7. "Reasons for unmet need for family planning, with attention to the measurement of fertility preferences: protocol for a multi-site cohort study." Reproductive Health I4, (23): I-II.

Mills, A., Brugha, R., Hanson, K. and McPake, 
B. 2002. "What can be done about the private health sector in low-income countries?" Bulletin of the World Health Organization 80, (4): 325-330.

$\mathrm{MOH}$ (Ministry of Health), Division of Family

Health, Family Planning Program [Kenya]. 20I8. National Family Planning Guidelines for Service Providers, 6th Edition. Nairobi, Kenya: RMHSU.

$\mathrm{MOH}$ (Ministry of Health) [Kenya]. 20I4. Health

Sector Human Resources Strategy 2014-2018. Nairobi: MOH/Republic of Kenya.

$\mathrm{MOH}$ (Ministry of Health) [Kenya]. 2013.

Kenya's Human Resources Commitments at the 2013 Third Global HRH Forum in Brazil. Nairobi: $\mathrm{MOH}$. http://www.health.go.ke/wpcontent/uploads/2015/09/Ministry\%20of\%20Heal th\%20Committment\%20revised.pdf (Accessed: April 2020).

Mutumba, M., Wekesa, E. and Stephenson, R. 2018. "Community influences on modern contraceptive use among young women in low and middle-income countries: a cross-sectional multi-country analysis." BMC Public Health 18 , (430): I-9.

NCPD (National Council for Population and

Development) and IRD (Institute for Research Development)/Macro Systems, Inc. 1989. Kenya Demographic and Health Survey 1989. Nairobi, Kenya and Maryland, USA: NCPD and IRD/Macro Systems, Inc.

Nonvignon, J. and Novignon, J. 2014. "Trend and determinants of contraceptive use among women of reproductive age in Ghana." African Population Studies 28, (2): 956-967.

Olakunde, B.O., Sam-Agudu, N.A., Patel, T.Y., Hunt, A.T., Buffington, A.M., Phebus, T.D., Onwasigwe, E., and Ezeanolue, E.E. 2019. "Uptake of permanent contraception among women in sub-Saharan Africa: a literature review of barriers and facilitators." Contraception 99, (4): 205-211.

Patouillard, E., Goodman, C.A., Hanson, K.G. and Mills, A.J. 2007. "Can working with the private for-profit sector improve utilization of quality health services by the poor? A systematic review of the literature." International Journal for Equity in Health 6, (17): I-II.

Population Council. 20I5. The Balanced

Counseling Strategy Plus: A Toolkit for Family Planning Service Providers Working in High STI/HIV Prevalence Settings. New York: Population Council.

Schivone, G.B. and Blumenthal, P.D. 2016.

"Contraception in the developing world: special considerations." Seminars in Reproductive Medicine 34, (03): I68-174.

Tessema, G.A., Gomersall, J.S., Mahmood,

M.A., and Laurence, C.O. 2016. "Factors determining quality of care in family planning services in Africa: a systematic review of mixed evidence." PLoS ONE II(II):e0165627, doi:I0.137I/journal.pone.0165627.

Tumlinson, K., Pence, B.W., Curtis, S.L., Marshall, S.W., and Speizer, I.S. 20I5. "Quality of care and contraceptive use in urban Kenya." International Perspectives on Sexual and Reproductive Health 4I, (2): 69-79.

Unumeri, G., Ishaku, S., Ahonsi, B. and Oginni, A. 2015. "Contraceptive use and its socioeconomic determinants among women in northeast and north-west regions of Nigeria: a comparative analysis.” African Population Studies 29, (2): $|85|-\mid 867$.

Walle, A and Woldie, M. 2017. "Clientcenteredness of family planning services in a resource limited setting." Ethiopian Journal of Health Science 27, (4): 373-382.

WHO/RHR (World Health Organization/

Department of Reproductive Health and Research) and (JHBSPH/CCP) (Johns Hopkins Bloomberg School of Public Health/Center for Communication Programs) Knowledge for Health Project. 2018. Family Planning: A Global Handbook for Providers (20I8 update). Baltimore and Geneva: CCP and WHO.

WHO/RHR (World Health Organization/

Department of Reproductive Health and Research). 2005. Decision-making Tool for Family Planning Clients and Providers. Geneva: WHO/RHR. 